\title{
Development of PCR Controller and Smart-Phone Application based on Bluetooth Communication
}

\author{
Jong-Dae Kim ${ }^{1,2}$, Chan-Young Park ${ }^{1,2, *}$, Jun Yeon ${ }^{1}$, Yu-Seop Kim ${ }^{1,2}$ \\ and Hye-Jeong Song ${ }^{1,2}$ \\ ${ }^{1}$ Dept. of Ubiquitous Computing, Hallym University, Korea \\ ${ }^{2}$ Bio-IT Research Center, Hallym University, Korea \\ \{ kimjd, cypark, mauver, yskim01, hjsong \}@hallym.ac.kr
}

\begin{abstract}
Polymerase Chain Reaction (PCR) is an amplification method of genetic materials and is used in almost every process that includes genetic material treatment. Conventional PCR machines control the process with a computer that is connected with a wire, limiting the mobility and requiring an expensive, high technological installation skill. This paper utilizes the wireless Bluetooth technology to resolve such problems, enabling the user to control the PCR process with a smartphone. The PCR machine is paired with a smartphone, and a protocol that can control the PCR machine is integrated in the smart phone. By controlling the PCR machine with a smartphone many advantages can be achieved from enabling PCR machines to be installed in an environment that does not provide wired networking, reduce the cost and improvement in convenience of control and mobility.
\end{abstract}

Keywords: PCR thermal cycler, Bluetooth, smart phone

\section{Introduction}

Polymerase Chain Reaction (PCR) is a common method to amplify DNA in molecular biology [1]. PCR thermocycler is a device controlling the temperature required for PCR by controlling the temperature of the chamber. PCR thermocycler should not only have the biochemical control function that controls the temperature of the chamber which in turn controls the temperature of the sample prepared for PCR [2], but also should have other system management functions such as data analysis and user interface [3-8]. Recently, even the simplest electronic devices require graphical user interface (GUI) and data accessibility through the internet, there is a high advantage when managing the system using a computer [9-10].

In most cases, the PCR thermocycler is controlled by machine with GUI. Recently, the PCR thermocycler is controlled by a host PC that is connected with a wire. However, this gives rise to an additional cost for the PC installation, and also decreases the efficiency since the PCR thermocycler and host PC is connected with a wire. In this research, a PCR thermocycler controller based on the Bluetooth function of smartphones is developed and its performance was evaluated to resolve such problems. In detail, an attempt to control the Stand alone form PCR machine by SPP (Serial Port Profile) using Bluetooth function of an android smartphone was made.

\footnotetext{
* Corresponding Author: cypark@hallym.ac.kr
} 
The smartphone was made to have a function where it can detect Bluetooth devices and a GUI that displays the results. The desired PCR thermocycler can be selected and connected to the smartphone. The user can input and save the protocol that will be used to control the PCR thermocycler in the smartphone, and can send the protocol to the PCR machine that will initiate the PCR process once connected. A method to prevent any problems that might occur when the smartphone is far out of the range to be connected via bluetooth with the PCR machine is also dealt with, stabilizing the overall system. As a result, the installation of a host PC was eliminated, increasing the efficiency space-wise. This paper introduces the research on PCR and Bluetooth in Section 2, explains the program developed in Section 3, and the conclusion is made in Section 4.

\section{Bluetooth Implements}

To use the bluetooth function in PCR control, the FB155BS model of Firmtech was used (in Figure 1). This Bluetooth module supports Bluetooth Specification 2.1 and uses the helical antenna type, then is installed in the PCR thermocycler with an antenna connected to the module.

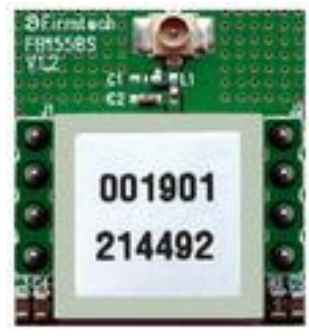

\section{Figure 1. FB155BS module from Firmtech}

In order to communicate with the Bluetooth device must use the Bluetooth profile. The Bluetooth profile is a specification regarding an aspect of Bluetooth-based wireless communication between devices. In order to use Bluetooth technology, a device must be compatible with the subset of Bluetooth profiles necessary to use the desired services. A Bluetooth profile resides on top of the Bluetooth Core Specification and (optionally) additional protocols. While the profile may use certain features of the core specification, specific versions of profiles are rarely tied to specific versions of the core specification [14]. There are many types of Bluetooth as Table 1, in order to communicate with PCR Bluetooth Module, we use the SPP (Serial Port Profile).

Most android smartphones already have Bluetooth functions as a default. Galaxy Tap 10.1 from Samsung electronics was chosen in this paper. For the smartphone to detect the SPP Bluetooth module inside the PCR machine, the UUID (Universally unique identifier) of the smartphone Bluetooth has to be set into SPP, then it can be connected with the PCR machine via Bluetooth. After connected, the following messages shown in Table 2 will be displayed in the GUI of the smartphone, enabling the users to control the PCR thermocycler. 
Table 1. Bluetooth profile list

\begin{tabular}{|c|c|}
\hline Profile & Description \\
\hline Device ID Profile (DIP) & $\begin{array}{c}\text { This allows a device to be identified above } \\
\text { and beyond the limitations of the Device } \\
\text { Class already available in Bluetooth. }\end{array}$ \\
\hline Dial-up Networking Profile (DUN) & $\begin{array}{c}\text { This provides a standard to access the } \\
\text { Internet and other dial-up services over } \\
\text { Bluetooth. }\end{array}$ \\
\hline File Transfer Profile (FTP) & $\begin{array}{c}\text { Provides the capability to browse, } \\
\text { manipulate and transfer objects (files and } \\
\text { folders) in an object store (file system) of } \\
\text { another system. }\end{array}$ \\
\hline Human Interface Device Profile & $\begin{array}{c}\text { Provides support for devices such as mice, } \\
\text { joysticks, keyboards, as well as sometimes } \\
\text { providing support for simple buttons and } \\
\text { indicators on other types of devices. }\end{array}$ \\
\hline Serial Port Profile (SPP) & $\begin{array}{c}\text { It emulates a serial cable to provide a } \\
\text { simple substitute for existing RS-232, } \\
\text { including the familiar control signals. }\end{array}$ \\
\hline
\end{tabular}

Table 2 shows the messages shown in the GUI of the smartphone and its functions. 'STATUS' message receives the status of the PCR thermocycler every 0.1 seconds after it is connected to the smartphone. The reply of this message includes the current temperature and TASK of the PCR machine, and the remaining time. If there is a malfunction in the PCR thermocycler, or if the TASK value that increases the temperature is too high, the PCR protocol stops and the error value is transmitted to the smartphone. 'TASK_WRITE' message and 'TASK_END' message determine the duration and temperature for each steps of the PCR protocol. 'GO' message orders the PCR thermocycler to start with the given TASK values. 'STOP' message can end the PCR protocol manually.

Table 2. Function of each message

\begin{tabular}{|c|c|}
\hline Message & Function \\
\hline STATUS & Get status of PCR \\
\hline TASK_WRITE & Write TASK to PCR machine \\
\hline TASK_END & Notify user that TASK is successfully written \\
\hline GO & Start PCR protocol \\
\hline STOP & Stop PCR protocol \\
\hline
\end{tabular}

The interaction diagram between the PCR controller and smartphone is shown in Figure 2. 


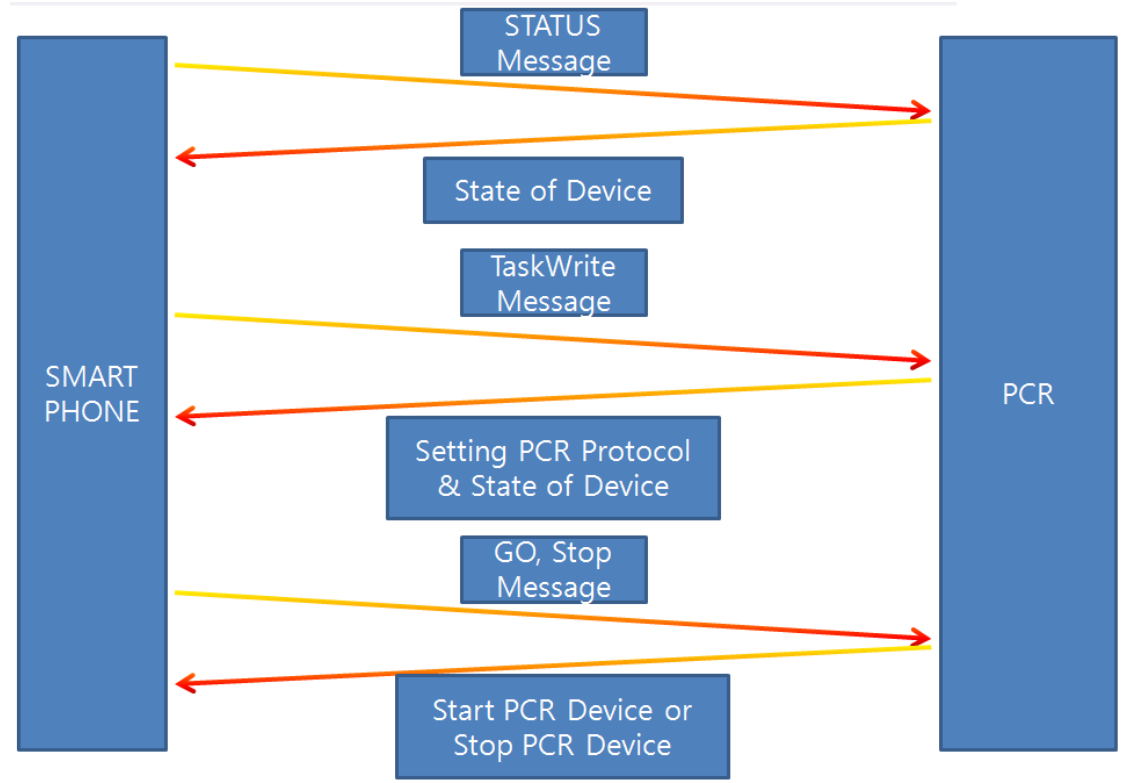

Figure 2. Interaction between PCR controller and smartphone

\section{Realization}

The PCR machine used in the experiment is the MyPCR from Labasis Co., which is controlled by a PC that is connected with a USB (in Figure 3 left side). Since this PCR machine is developed in a standalone form, once the RUN order is transmitted to MyPCR form the host, it becomes an independent machine, and therefore can continue the PCR protocol without any trouble even is the connection is interfered. A PCR thermocycler that can be controlled via Bluetooth was made by installing the FB155BS Bluetooth module from Frimtech co. in MyPCR. In Figure 3, right side is the Bluetooth installed version. We can see the outside antenna in top of the system. The Bluetooth version has removed the USB function.

A controller to control MyPCR was developed on a android platform by using a Bluetooth API supported by android. The controller should consist of three parts: a part connecting with the PCR machine, a part to set the TASK manually, and a part that shows the current status of the PCR.

Figure 4 illustrates the smartphone main UI of the PCR control application. Main UI will show you what the status of the LED device of PCR and the remaining time of the protocol name and protocol, which has been read with the Protocol button. Also, it displays in real time the temperature of the Chamber and the PCR connected serial number, the temperature of the LID Heater. Preheat value when the PCR is running, that can set the temperature value of the LID Heater. The user can create their own TASK and save them in a list by pressing the 'Protocol' button, and also choose which TASK to send from the list. When a protocol (TASK) is selected, the 'Start' button activates. The Bluetooth devices are detected by pressing the 'Start' button, and the protocol (TASK) is only transmitted when MyPCR is selected from the list of devices. 


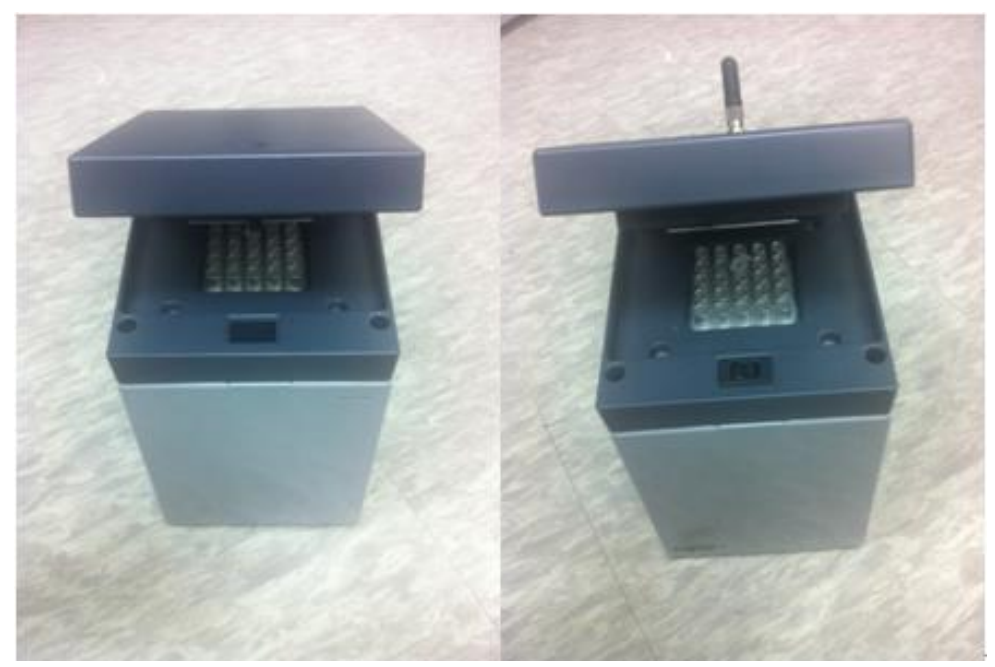

Figure 3. MyPCR USB version(left), MyPCR Bluetooth version(right)

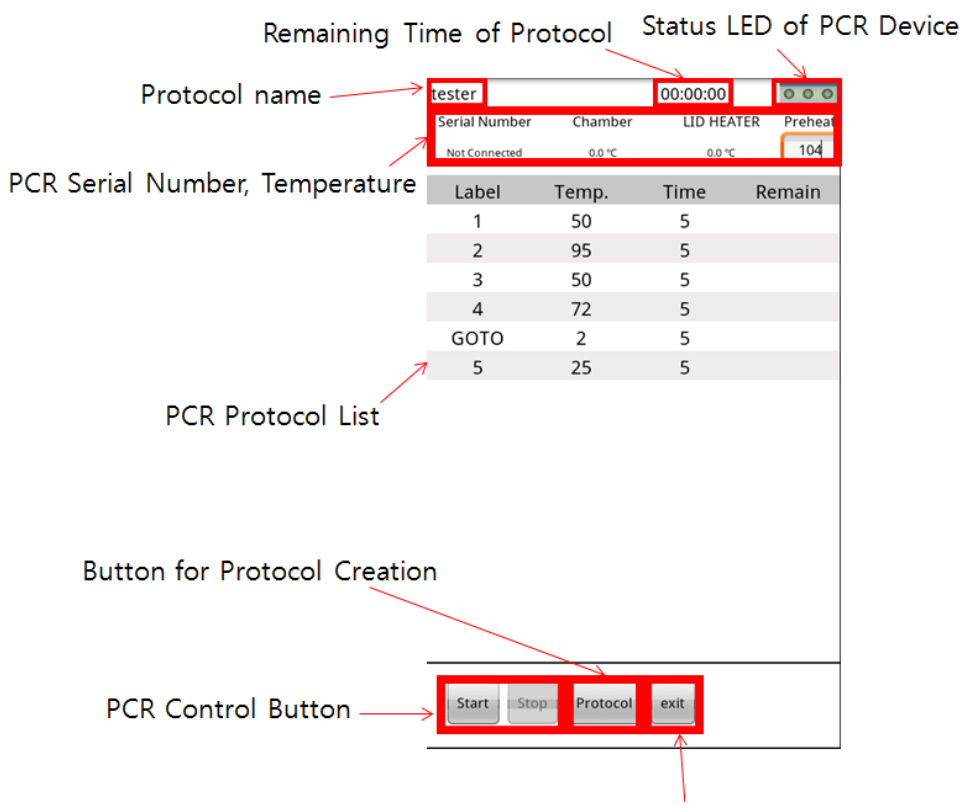

Exit Program

Figure 4. Android smartphone Main UI

Figure 5 is a UI to create a protocol for communicating with MyPCR. You can set whether or not the operation temperature for a few seconds in order to Label MyPCR. GOTO Label is a means to move only the value of the Time to Label which is set to Temp. Can enter a value for the temperature in the range that can be controlled by MyPCR, and setting a value of 0 Time, an infinite amount of time to work. 


\begin{tabular}{|c|c|c|c|c|}
\hline \multicolumn{5}{|c|}{ tester Protocol Setting } \\
\hline Label & Temp & Time & & \\
\hline 6 & & & Add & Gото \\
\hline \multicolumn{2}{|c|}{ Label } & Temp & & Time \\
\hline \multicolumn{2}{|c|}{1} & 50 & & 5 \\
\hline \multicolumn{2}{|c|}{2} & 95 & & 5 \\
\hline \multicolumn{2}{|c|}{3} & 50 & & 5 \\
\hline \multicolumn{2}{|c|}{4} & 72 & & 5 \\
\hline \multicolumn{2}{|c|}{ GOTO } & 2 & & 5 \\
\hline \multicolumn{2}{|c|}{5} & 25 & & 5 \\
\hline
\end{tabular}

Figure 5. Android smartphone Protocol Generation UI

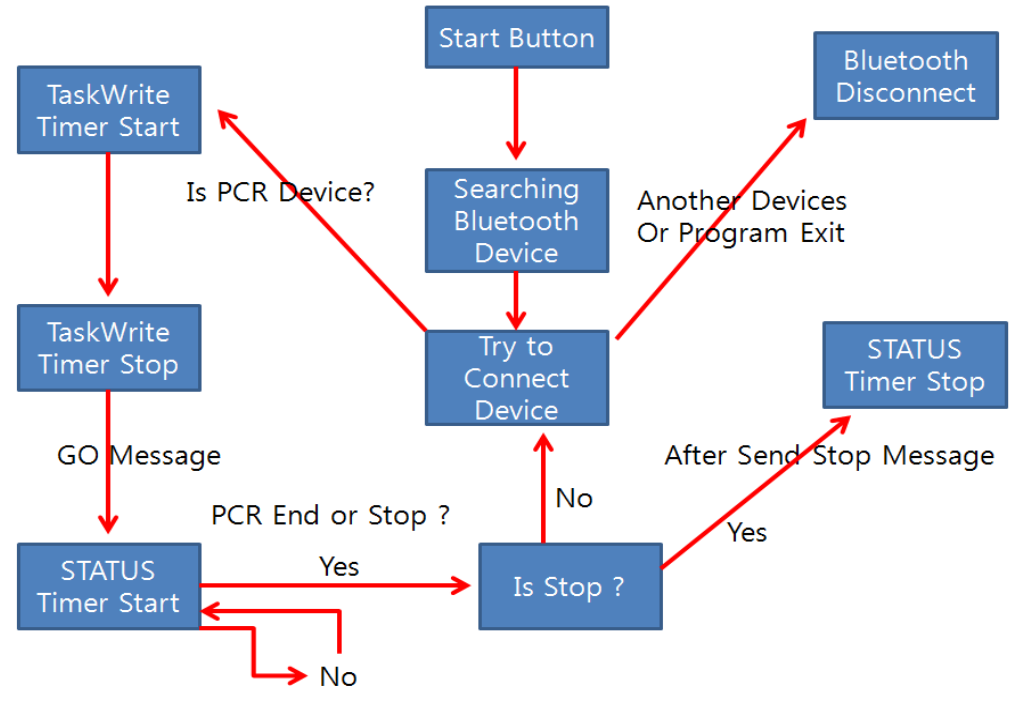

Figure 6. Flow chart of android smartphone program

The flow chart of the PCR control application is shown in Figure 6. The value of TASK is transmitted to the PCR thermocycler every 0.1 seconds, and is set so that no other device can be connected during the transmission. After the PCR starts, any other messages except for 'STATUS' message and 'STOP' message will be read as 'STATUS' message when input, and will send the status of the PCR machine to the smartphone. Since Bluetooth function has range limitation being a wireless network, the PCR machine will disconnect form the smartphone. However, the PCR thermocycler runs according to the independently saved 
protocol, the PCR will stay in a regular or irregular stop status. When reconnected, the result of the last execution will be sent to the smartphone to notify the user of its status.

\section{Conclusion}

This paper developed a bluetooth based smartphone PCR controller with the goal to decrease the installation cost by elimination the need of a host PC, and to increase the convenience of installation by using wireless network. This will also help manage the PCR protocol easily, and enable the installation of PCR machines in laboratories with insufficient space. Control of multiple PCR thermocyclers can also be achieved with less effort, if integrating a function that can read the protocols from the PCR machine to the smartphone and a function that can monitor multiple PCR machines simultaneously in the smartphone application. In addition, there is no need to produce a PCR for each version if implemented so as to be able to control any of the current Bluetooth and USB, which is very convenient. If this can be realized, the space and cost problems that might arise when purchasing and installing PCR thermocyclers can be resolved.

\section{Acknowledgments}

The research was supported by Basic Science Research Program through the National Research Foundation of Korea(NRF) funded by the Ministry of Education, Science and Technology(2012R1A1A2040381) and Hallym University Research Fund, 2012 (HRF201209-027).

\section{References}

[1] C. R. Newton and A. Graham, PCR, BIOS Scientific Publishers Limited, United Kingdom, (1994).

[2] William J. Benett, Livermore, CA(US); James B. Richards, Danvile, CA(US), "PCR thermocycler", patent No.: US 6,503,750 B1, (2003), pp. 5-7.

[3] P. C. Chena, "Titer-plate formatted continuous flow thermal reactors: Design and performance of a nanoliter reactor", Sensors and Actuators B, vol. 149, (2010), pp. 291-300.

[4] K. H. Chung, Y. H. Choi, and M.Y. Jung, "Natural Convection PCR in a Disposable Polymer Chip", IEEE SENSORS Conference, (2009).

[5] T. -M. Heieh, "Enhancement of thermal uniformity for a micro thermal cycler and its application for polymerase chain reaction", Sensors and Actuators B, vol. 130, (2008), pp. 848-856.

[6] K. Shen, X. Chen, M. Guo and J. Cheng, "A microchip-based PCR device using flexible printed circuit technology", Sensors and Actuators B, vol. 105, (2005), pp. 251-258.

[7] Q. Zou, "Micro-assembled multi-chamber thermal cycler for low-cost reaction chip thermal multiplexing", Sensors and Actuators A, vol. 102, (2002), pp. 114-121.

[8] J. -H. Wang, "A miniaturized quantitative polymerase chain reaction system for DNA amplification and detection", Sensors and Actuators B, vol. 141, (2009), pp. 329-337.

[9] J. D. Kim, Y. U. Lee and S. Kim, "Efficient hardware-software partitioning for a digital dental X-ray system", IEICE Trans. Fundamentals, vol. E86-A, no. 4, (2003) pp. 859-865.

[10] C. E. William, "User interface design for the engineer", Electro/96 International. Conference Proceedings. Combined, vol, (1994), pp. 415-419.

[11] S. -H. Whang, I. -H. Jang and K. -b. Sim, "Self-Organizable Bluetooth Network for Distributed Robot System", Proceedings of KFIS Spring Conference, vol. 14, no.1, (2004).

[12] C. -Y. Park, J. -D. Kim, Y. -S. Kim, H. -J. Song, J. -M. Kim and J. Kim, "Cost Reduction of PCR Thermal Cycler", International Journal of Multimedia and Ubiquitous Engineering, vol. 7, no. 2, (2012), pp. 389-394.

[13] FB155BS Datasheet, http://www.firmtect.co.kr/02download/datasheet/bluetooth/embed/ FB155/FB155_UserGuide_Kor.pdf.

[14] Bluetooth profile, http://en.wikipedia.org/wiki/Bluetooth_profile. 


\section{Authors}

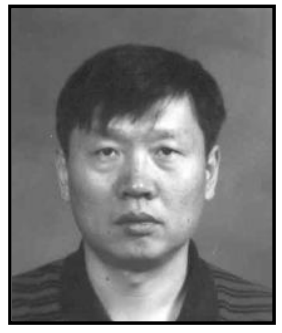

Jong-Dae Kim received his M.S. and the Ph.D. degrees in Electrical Engineering from Korea Advanced Institute of Science and Technology, Seoul, Korea, in 1984 and 1990, respectively. He worked for Samsung Electronics from 1988 to 2000 as an electrical engineer. He is a Professor in Department of Ubiquitous Computing, Hallym University. His recent interests focus on biomedical system and bioinformatics.

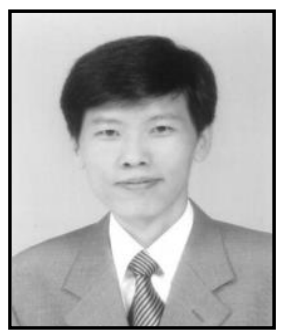

Chan-Young Par received his B.S. from Seoul National University in 1987 and the M.S. and the Ph.D. degree from Korea Advanced Institute of Science and Technology in 1989 and 1995, respectively. From 1991 to 1999, he worked for Samsung Electronics. He is currently a Professor in the Department of Ubiquitous Computing of Hallym University, Korea. His research interests are in Bio-IT convergence, Intelligent Transportation System and sensor networks.

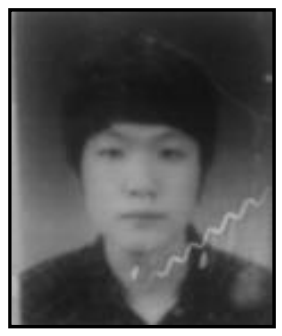

Jun-Yeon is enrolled in a fourth-year student in Ubiquitous Computing major in Hallym University. He currently studies for a master's degree in Computer Engineering at Hallym University. His recent interests focus on embedded system and computing communication system.

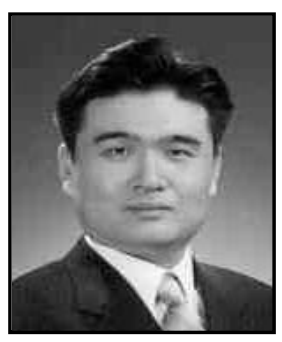

Yu-Seop Kim received his Ph.D. degree in Computer Engineering from Seoul National University. He is currently a Professor in the Department of Ubiquitous Computing at Hallym University, South Korea. His research interests are in the areas of bioinformatics, computational intelligence and natural language processing.

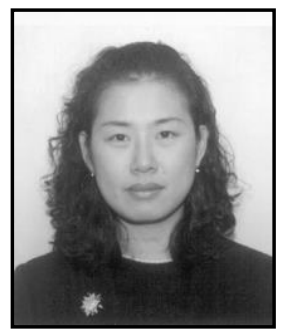

Hye-Jeong Song received her Ph.D. degree in Computer Engineering from Hallym University. He is a Professor in Department of Ubiquitous Computing, Hallym University. His recent interests focus on biomedical system and bioinformatics. 